\title{
On Mobility Analysis of Functional Sites from Time Lapse Microscopic Image Sequences of Living Cell Nucleus $^{\star}$
}

Lopamudra Mukherjee $^{1}$, Vikas Singh ${ }^{1}$, Jinhui Xu${ }^{1}$, Kishore S. Malyavantham ${ }^{2}$, and Ronald Berezney ${ }^{2}$

${ }^{1}$ Department of Computer Science and Engineering, State University of New York at Buffalo

$\{1 \mathrm{~m} 37$, vsingh, jinhui\}@cse.buffalo.edu

2 Department of Biological Sciences, State University of New York at Buffalo

$\{$ ksm4, berezney\}@buffalo.edu

\begin{abstract}
Recent research in biology has indicated correlations between the movement patterns of functional sites (such as replication sites in DNA) and zones of genetic activity within a nucleus. A detailed study and analysis of the motion dynamics of these sites can reveal an interesting insight into their role in DNA replication and function. In this paper, we propose a suite of novel techniques to determine, analyze, and interpret the mobility patterns of functional sites. Our algorithms are based on interesting ideas from theoretical computer science and database theory and provide for the first time the tools to interpret the seemingly stochastic motion patterns of the functional sites within the nucleus in terms of a set of tractable 'patterns' which can then be analyzed to understand their biological significance.
\end{abstract}

\section{Introduction}

DNA replication is the process of copying a DNA strand in a cell prior to division and is among the most important processes inside the mammalian cell nucleus. These replication (or synthesis) processes occur at so called replication sites (RS, for short). The study of the basic properties of these sites is fascinating not only because it helps interpret the fundamental functions of a cell but may also lead to understanding variations between a cell's healthy and diseased states (such as cancer). Recent developments in microscopic imaging techniques[1|2] have therefore focused on imaging RS in action in order to obtain a better understanding of their dynamics. The last few years in particular have seen several notable advances in live nuclear microscopic imaging and the development of associated software modules for image processing and analysis. This has been complemented by simultaneous developments in the usage of fluorescent proteins for staining these sites before imaging for better identification and visualization. These advances collectively have provided biologists for the first time a view of the spatial location, movement, and other behavioral aspects of RS within living cells in real time.

\footnotetext{
* This research was supported by NSF award CCF-0546509 and NIH grant GM 072131-23.
} 
From a biologist's perspective, RS exhibit an extremely interesting characteristic in that they are constantly in motion. This interest stems partly from observations reported in recent literature[3] which show a significantly faster movement of the sites encompassing the euchromatin DNA compared to those around the hetrochromatin DNA. Because it is well known that euchromatin is composed mostly of actively expressing genes whereas heterochromatin is largely inactive, the observed motion behavior strongly suggests a deeper connection between the sites' mobility patterns and the process of gene expression. These relationships can only be explored if the motion dynamics of these sites are well understood. The focus of our work is the design of algorithms that enables us to determine, study, and interpret these mobility patterns.

A closer analysis of the problem reveals several potential complications. For instance, a visual evaluation of the data suggests that the sites undergo an almost random motion between consecutive image frames. A temporal tracking algorithm adds little extra information towards any discernible patterns of movement except that it yields the sites' individual motion magnitudes from one image frame to the next. Moreover, details about the kinds of motion dynamics these sites undergo during various cell phases are not yet known. This makes the mathematical formulation of the problem challenging because we know neither the behavior of an ideal system (such as human vision/cognition) on this input nor the kind of patterns we would like to determine. Part of the reason that human vision also fails to extract any useful output from such data is that any intrinsic motion patterns are apparently irrecoverable from an overwhelming global to-and-fro-motion the sites exhibit. Given these difficulties, our objective is to search for some "method in madness" in an effort to represent the seemingly stochastic motion as a combination of a set of tractable functions.

In this paper, we propose novel techniques to address the problems discussed above in an effort to study the mobility properties of the RS. We approach this goal with a two-fold objective. The first question we try to answer is whether given a set of point 1 apparently undergoing haphazard motion, are there small subsets of points that show similar degree of motion. To answer this question, we propose a technique based on high dimensional point set clustering for grouping sites based on their mobility properties. We then illustrate how we employ this idea to identify spatial patterns by splitting the nucleus into various almost-independent mobility zones. Our second independent goal is to identify sets of points that seem to be moving together, as if linked by a slightly deformable chain (sub-structure identification). Observe that the true motion pattern, say $P$, the sites undergo is unknown. Nonetheless, it is expected that if a group of points are indeed moving rigidly as a substructure, their mobility (high or low) should be same for each point in that group. Therefore, to evaluate our algorithms we compare the results obtained using our two techniques and observe that there is a high degree of territorial overlap between the rigid point sub-sets generated in the second step and the mobility zones determined from the first; further, the patterns determined are consistent with expert opinion.

\footnotetext{
${ }^{1}$ In the remainder of this paper, we will use the terms sites $(R S)$ and points interchangeably depending on the biological or geometric context of the discussion.
} 


\section{Method}

\subsection{Temporal Tracking of Replication Sites (RS)}

The displacement (motion magnitude) of RS between consecutive image frames in the sequence can broadly be classified into two separate types of movement. The first and arguably the more interesting type of movement is due to the individual motion of the sites. The second type of movement can be attributed to a nominal displacement of the complete nucleus from one image frame to the next. Before attempting to address (and determine) the patterns of movements of individual sites, we employ a simple preprocessing step to correct for the global displacement (an isometric transformation owing to the second type of movement), that the nucleus undergoes. This procedure also provides temporal tracking information that yields the point to point correspondences from a pair of images. While such a process is by no means sufficient towards our ultimate goal of motion analysis because it yields no information about the patterns of movement, nonetheless, it serves as a useful first step. For this purpose, we employ a simple technique proposed by Cho and Mount[4] to calculate an alignment between the pair of point sets representing RS in consecutive image frames. While the theoretical performance analysis of the algorithm guarantees an approximation ratio of 3 (alignment will be no worse than three times the unknown optimal alignment under Hausdorff distance measure), we observe that such an analysis is quite conservative. In practice, the technique performs favorably by calculating a transformation (rotation matrix, $\mathbf{R}$, and a translation vector, $\mathbf{t}$ ), that aligns the two point sets quite well. Once this alignment has been determined, we calculate point to point correspondences by using a combination of bipartite matching and rank-maximal matching coupled with certain assumptions about a neighborhood of motion. The size of the neighborhood is chosen based on the temporal resolution of the image sequence (usually, about 2 seconds) and the maximal to-and-fro motion a site can be expected to undergo within this time period. The results are then manually verified to ensure whether such a procedure returns accurate correspondences. In general, we obtain an accuracy of about $90 \%$ which is comparable to those reported in recent literature [5].

\subsection{Determining Mobility Zones}

To investigate the mobility properties of the sites, our main idea is to to zone (or cluster) the sites based on their motion magnitude and then use this information to spatially partition the image into mobility coded regions. We proceed as follows. The number of mobility zones $(k)$ and the 'window' of time to be considered for determining the non-uniform motion of the sites are assumed given. Here, a fixed time window consists of $d$ discrete intervals or image frames. The parameters $k$ and $d$ are then used to create (and populate) a high dimensional feature space $S$, where $S \in \mathfrak{R}^{d}$ if the left end of the time window is placed on (but not including) time point $t_{0} . S$ is populated by making use of the tracking information obtained in $\$ 2.1$. Consider a graph, $G$, induced by the correspondence information determined by the temporal tracking algorithm. $G$ has $d$ 'levels' (or parts) where points in the image frame at time point, $t_{j}$, are represented as nodes in $G$ at level $j$, say $G_{j}$. The information that a point $p_{i}^{j}$ in the image at $t_{j}$ 
corresponds to a point $p_{k}^{j+1}$ can be easily represented in $G$ by introducing a directed edge between the nodes corresponding to $p_{i}^{j}$ and $p_{k}^{j+1}$. The weight of an edge from a node $n_{i}^{j} \in G_{j}$ to $n_{k}^{j+1} \in G_{j+1}$ can be calculated by considering the distance traveled by the point $p_{i}^{j}$ from time point, $t_{j}$ to $t_{j+1} \operatorname{given}$ by dist $(\cdot, \cdot)$ denoting the $L_{2}$ distance between $p_{i}^{j}$ and $p_{k}^{j+1}$. Our feature space, $S$, can then be easily populated by considering each unique (not necessarily node disjoint) correspondence path from a node in $G_{1}$ to a node in $G_{d}$. The inhomogenous representation of a point in $S$ can then be calculated by considering the edge weights (in order) of its corresponding correspondence path. Note that the those image points that could not be tracked will not have a complete correspondence path from $G_{1}$ to $G_{d}$ and thus will not be represented in $S$.

Our purpose now is to determine $k$ spatial clusters in $S$; each cluster will represent a 'mobility zone' and an inverse transformation on $S$ will yield information about the mobility patterns of individual points in the input images. We based our algorithm on a hierarchical clustering technique known as Agglomerative clustering [6]. Hierarchical clustering algorithms work in arbitrary number of dimensions and group items into a hierarchy of clusters relying on distance measures to determine similarity between clusters. This cost function is then minimized in an optimization framework. Agglomerative clustering in particular works by considering each entity as an individual cluster, and then pairs of items are repeatedly merged until the total number of clusters in $S$ is exactly $k$ (similar to Kruskal's Minimum Spanning Tree algorithm). We employ a special type of agglomerative hierarchical clustering called CURE (Clustering Using Representatives) [7] that has a running time complexity of $O\left(n^{2}\right)$. It performs reliably, is robust with respect to the value of $k$, and produces high quality clusters even in the presence of outliers. Once the clustering is done, we assign to each cluster a unique 'color tag' as an identifier. An inverse transformation is then applied on $S$; this yields a color tag for each correspondence path in $G$. Clearly, if the points corresponding to two correspondence paths, $P_{1}$ and $P_{2}$, were assigned the same color (members of the same cluster in $S$ ), the colors tags for $P_{1}$ and $P_{2}$ will also be the same. The color tags of the correspondence paths are then transferred back to the sets of RS in the input image sequence. Observe that the clustering process uses only the motion parameters and is independent of the spatial distribution of the points. However, using the clustering information, we can now analyze the sites' spatial properties in context of the motion they undergo and investigate if any interdependencies can be determined. We do this as follows. First, we partition the nucleus using Voronoi diagrams [8], dividing it into spatial regions of influence based on the distribution of sites. We then make use of the sites' color tags (obtained via an inverse mapping on $S$ ) to merge adjacent polygons having the same color tag to obtain larger cells obtaining a chromatic Voronoi Diagram. This final step provides us with a very useful tool for analyzing the spatial relationships of the RS. Notice that larger cells in the chromatic voronoi diagram are in fact a contiguous mobility sub-region and indicate a sub-structure of RS undergoing similar motion over the time window. By analyzing the chromatic voronoi diagrams for an entire sequence (all $d$ image frames in a sequence), we can not only assess the reliability of the scheme (the partitioning should be fairly continuous over the given time window and should not change abruptly from one image frame to the next), but also analyze the motion dynamics of a given sequence of RS (see Fig. 1 for an illustration). 


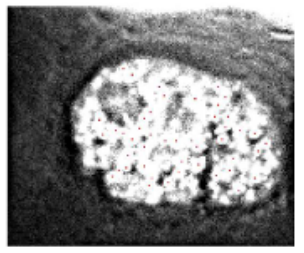

(a)

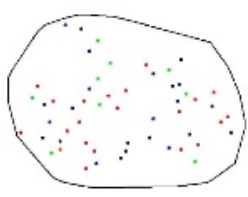

(b)

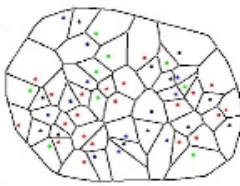

(c)

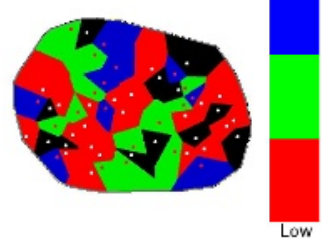

$(d)$

Fig. 1. Illustration of (a) original cell nuclear image, (b) sites colored according to mobility based clustering, (c) Voronoi Partitions of the sites, (d) contiguous mobility zones determined by merging adjacent voronoi cells with the same color tag. The color scheme used to denote the zones is denoted by the color bar on the right. Regions containing sites which could not be tracked (about $10 \%)$ are shown in black.

Remark: Our attempts at obtaining an equivalent clustering solution in $1 D$ using the mean values of the feature vectors yielded significantly different results. We suspect that motion variability exhibited by a RS in $d$ frames is not sufficiently captured by its mean velocity alone.

\subsection{Determining Rigid Substructures}

Our strategy here is to determine subsets of points that approximately retain their substructure from one image frame to the next (and over the sequence). This is a key difference to the previous motion-based clustering scheme because substructure similarity of the form we address here is independent of motion (displacement transformations such as translations and rotations). Similarity and agreement in the results of these independent techniques $\sqrt{2}$ on the same data set indicates a fair likelihood of accuracy in the absence of any gold standard data.

In recent literature, there has been a flurry of activity aimed at utilizing structural pattern identification techniques to study common substructures of proteins in bioinformatics research [9]. The common feather in most of these approaches is the representation of proteins as geometric graphs. Each node in the graph represents a 'unit' (or atom) in the protein and has real world coordinate values; the edges between the nodes represent the inter-atomic bonding. Each technique then tries to calculate a similarity measure between these graphs (or trees). Adapting these techniques to our problem, however, is rather difficult. because no apparent 'links' between the RS are known. Of course, an alternative is to look for patterns for every possible grouping of points. While this may work for a small number of points, our datasets are typically large. This would require a substantial amount of computation time making it practically infeasible.

Taking these issues into account, the approach we adopt is as follows. Consider two point sets, $P_{1}$ and $P_{2}$, corresponding to RS in consecutive image frames. For every triplet of point in the first set, say $\left(p_{i}, p_{j}, p_{k}\right)$, a create a point $p_{i j k}^{\prime}$ in a search space, $S$, such that

\footnotetext{
2 The previous technique performs clustering in a feature space based solely on motion while the second technique will consider 'spatial structures' and ignore motion.
} 
$p_{i j k}^{\prime}=\left(d_{i j}, d_{i k}, d_{k j}\right)$ where $d_{i j}=\operatorname{dist}\left(p_{i}, p_{j}\right), d_{i k}=\operatorname{dist}\left(p_{i}, p_{k}\right), d_{k j}=\operatorname{dist}\left(p_{k}, p_{j}\right)$ and $d_{i j} \geq d_{i k} \geq d_{k j}$ (dist $(\cdot, \cdot)$ denotes the $L_{2}$ distance). Thus, we obtain two sets of points, $P_{1}^{\prime}$ and $P_{2}^{\prime}$ in $S$, each comprising of no more than $n^{3}$ points (if $\max \left(\left|P_{1}\right|,\left|P_{2}\right|\right)=n$ ). The process is useful because it converts our substructure isomorphism determination problem into a point location problem as we shall discuss now. Consider a triplet of points that did not undergo any structural change from the first time point to the next. The reason why this transformation to a point location problem works is that the triplet of points at the time point $t_{P_{1}}$, say, $\left(u_{i}, u_{j}, u_{k}\right)$, and the triplet of points at time point $t_{P_{2}}$, say, $\left(v_{x}, v_{y}, v_{z}\right)$, will map to the same point in $S$. In other words, the notion of a $\varepsilon$-neighborhood in $S$ is analogous to a quasi isomorphism between triplets of RS in consecutive image frames (where $\varepsilon$ is a small constant). To exploit this neighborhood information, we make use of range-search trees [8] for point location queries in $S$. However, since the transformation to $S$ loses all location information of triplets of RS, we check potentially isomorphic structures against the tracking information calculated in $\$ 2.1$ and verify if a given pair of two triplet of points are isomorphic under some rigid transformation $T$. Then, we gradually increase the sets of likely matches to include more than three points at a time. This strategy allows us to avoid paying an exorbitant cost associated with checking all combinations of $\left(\begin{array}{l}n \\ r\end{array}\right)$ point sets in consecutive image frames (where $r \geq 3$ ). Finally, the process yields isomorphic substructures in consecutive image frames.

\section{Results}

Our algorithms were implemented in $\mathrm{C}^{++}$using CGAL and LEDA on a machine running GNU/Linux. Evaluations were performed on 15 temporal data-sets $(2 D+$ time $)$, each consisting of about 10 image frames taken 2 seconds apart. For simplicity of presentation, we will discuss results for each subsection in the order they appeared in the paper. Due to space limitations, we will omit details about the preprocessing phase. We will try to highlight our main observations here using a few datasets as illustrative examples instead of discussing results for each set individually.

Motion based high dimensional clustering: The correspondence information from $\$ 2.1$ was obtained and our algorithms described in $\$ 2.2$ were used to determine clusters using user provided parameter $k$ and $d$ based on the sites' motion magnitudes. The color tags of each of the $k$ motion clusters were then used to 'tag' its member RS. A visualization of these color-coded sites in real space revealed a rather interesting property. We found that a significant number of spatially proximate sites (in real space) were allocated to the same cluster in the high dimensional feature space. Further, a partition of the nucleus (based on Voronoi Diagrams) showed a number of adjacent cells joining together to form contiguous mobility zones. This observation indicates that close-together sites show a similar degree of motion as previously illustrated in Fig.11,

The robustness of our algorithm was further evaluated by varying the two input parameters: (1) value of $k$ (number of clusters), (b) length of time window (dimensionality of the feature space, $d$ ). (1) varying the value of $k$. We noticed that the spatial correlations of sites that belong to the same mobility cluster in feature space are preserved 
under varying values of $k$. In Figs.2(a)-(c), we illustrate the results of color partitioning of the nucleus (represented by the first image frame in the sequence) based on mobility clustering for different $k$ values. Observe that between Fig. 2 (a) and 2 (b), the overall partitioning schema remains approximately constant. However, based on the number of clusters desired (the chosen value of $k$ ), the higher mobility zone in (a) was upgraded to being the highest mobility zone in (b) whereas the lower mobility zone of (a) showed more variation in movement splitting up into two zones. A similar phenomena is seen when we increase $k$ from 3 to 4 in Figs. 2 (b) and (c). This trend conveys a strong sense

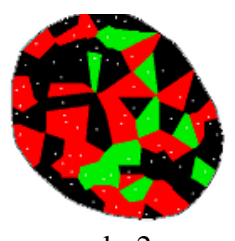

$\mathrm{k}=2$
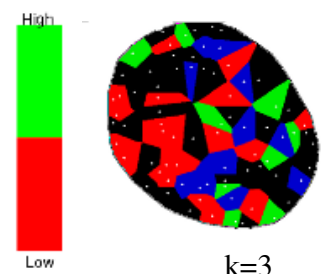

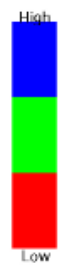

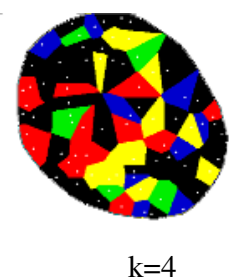

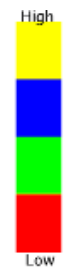

Fig. 2. Nucleus of a cell showing varying number of mobility zone

in that the clustering technique is robust to the value of $k$. Therefore, one can increase the value of $k$ if a more vivid detail is desired (more colors in the partition). (2) increasing the length of the time window. We observed that a lengthier time window (higher dimensionality, $d$, of feature space) does not have a detrimental effect on the performance of the clustering. In fact, spatial relationships of the clusters determined for several different choices of time windows were very similar (for a fixed $k$ value). We illustrate these results in Figs. 3(a)-(c). The only deterioration we could notice was that as the length of the time window became progressively larger, the number of sites that could not be tracked also increased (showing more black regions in Figs. 3 (a)-(c)). We suspect that this might be as a consequence of inaccuracies in the temporal tracking (preprocessing) phase. (3) Overall quality of clusters. The overall quality of mobility

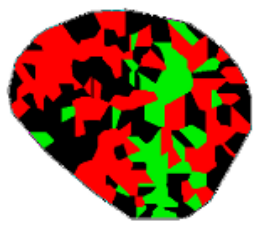

$\mathrm{d}=4$

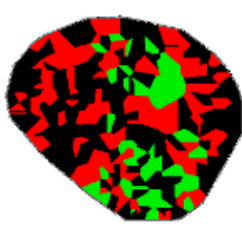

$\mathrm{d}=6$

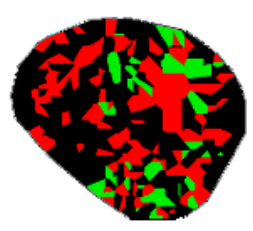

$\mathrm{d}=8$

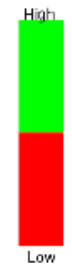

Low

Fig. 3. Nucleus of a cell showing mobility zones determined from time point 0 to 4 , time point 0 to 6 and time point 0 to 8

based clustering was observed to be quite good (calculated using metrics suggested in [7] and other simple measures such as geometric standard deviations with respect to the centroid of the cluster). While we will avoid a detailed discussion on this topic due to lack of space but would like to point out that the standard deviation of the clusters remained relatively constant as a function of an increase in the length of the time window 
(related to (2) above). However, we noticed an almost linear relationship between the compactness and the value of $k$ chosen (related to (1) above).

Rigid substructure determination: The algorithm described in $\$ 2.3$ were used to determine rigid substructures using parameters $d$ and a maximum allowable deformation ع. Fig. 4 (a) shows some of rigid substructures obtained for a particular sequence of images where $d=5$ (time point 0 to 5). Rigid substructures of points identified by our technique are illustrated as a minimum spanning tree. Small deformative changes in the rigid substructures across the time sequence manifest as variations in the spanning tree structure from one time point to the next (see Fig.4). For example, in 4 (c) (which shows an enlarged view of one of the rigid substructures in Fig. 4 (a) with correspondence of points), point labeled $j$ was connected to point $i$ in time point 0 but its corresponding point $j^{\prime \prime}$ in time point 3 was connected to point $h^{\prime \prime}$, indicating that it moved closer to point $h$ in the course of the time sequence, even though the overall substructure remained the same.

Agreement: To evaluate the agreement of the two techniques, we superimposed the rigid substructures obtained using the algorithms in $\$ 2.3$ 'on' the voronoi regions generated based on mobility based clustering. The results obtained on a sequence are shown in Fig. 4(a) and (c) whereas the superimposed image is shown in Fig. 4(b). In all datasets, we observed that the rigid substructures belong almost entirely to the same zone.

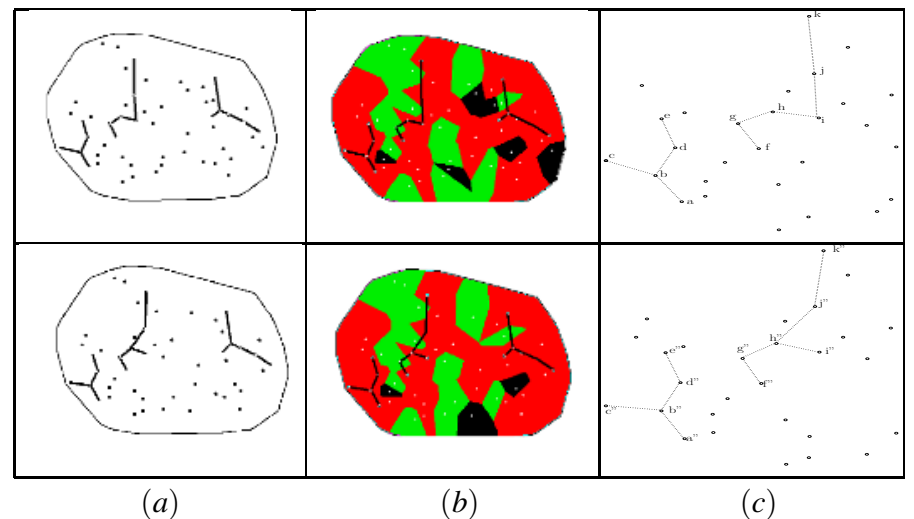

Fig. 4. A cell nucleus showing (a) rigid substructures (in a spanning tree form), (b) superimposed with mobility zones (color scheme same as in Fig. 3), (c) enlarged view of a sub-region for time point 0 (top) and time point 3 (bottom)

\section{Conclusions}

We have proposed several algorithms for determination and analysis of motion dynamics of functional sites. While the subproblems we address such as mobility based clustering and determination of quasi-isomorphic substructures are interesting in their own right, we are particularly excited by the 'motion patterns' in the movement of RS 
discovered by the technique. These results dispense (at least in part) the notion about the randomness of such movements. In fact, the strong correlation between spatial proximity and the degree of motion of the sites, coupled with presence of rigid substructures suggests that the movement of replication sites over small regions inside the nucleus are either inter-dependent or the sites are influenced equally by some unknown factor. Investigations are currently underway to understand and interpret these patterns in terms of nuclear replication, function and cell phases.

\section{References}

1. Rebollo, E., Gonzalez, C.: Time-lapse imaging of male meiosis by phase-contrast and fluorescence microscopy. Cell Biology and Biophysics 247 (2004) 77-87

2. Neumann, B., Held, M., Liebel, U., Erflea, H., Rogers, P., Pepperkok, R., Ellenberg, J.: Highthroughput RNAi screening by time-lapse imaging of live human cells. Nature Methods 3 (2006) 385-390

3. Berezney, R., Malyavantham, K.S., Pliss, A., Bhattacharya, S., Acharya, R.: Spatio-temporal dynamics of genomic organization and function in the mammalian cell nucleus. Advances in Enzyme Regulation 45 (2005) 17-26

4. Cho, M., Mount, D.: Improved approximation bounds for planar point pattern matching. In: Proc. Workshop on Algorithms and Data Structures (WADS). (2005) 432-443

5. Chen, X., Zhou, X., Wong, S.: Automated segmentation, classification, and tracking of cancer cell nuclei in time lapse microscopy. IEEE Transactions on Biomedical Engineering 53 (2006) 762-766

6. Jain, A.K., Murty, M.N., Flynn, P.J.: Data clustering: a review. ACM Comput. Surv. 31 (1999) 264-323

7. Guha, S., Rastogi, R., Shim, K.: CURE: An efficient clustering algorithm for large databases. In: In Proc. of ACM SIGMOD Int'l Conf. on Management of Data. (1998) 73-84

8. de Berg, M., Schwarzkopf, O., van Kreveld, M., Overmars, M.: Computational Geometry: Algorithms and Applications. Springer-Verlag (2000)

9. Wang, X., Wang, J., Shasha, D., Shapiro, B., Rigoutsos, I., Zhang, K.: Finding patterns in three dimensional graphs: Algorithms and applications to scientific data mining. IEEE Transactions on Knowledge and Data Engineering 14 (2002) 731-750 